\title{
Psychiatric Distress as a Common Risk Factor for Tinnitus and Joint Pain: A National Population-Based Survey
}

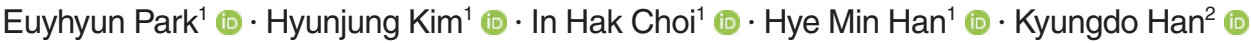 \\ Hak Hyun Jung ${ }^{1}$ (D) $\cdot$ Gi Jung $\operatorname{Im}^{1}$ (D) \\ ${ }^{1}$ Department of Otolaryngology-Head and Neck Surgery, Korea University College of Medicine, Seoul; \\ ${ }^{2}$ Department of Biostatistics, College of Medicine, The Catholic University of Korea, Seoul, Korea
}

Objectives. This study aimed to investigate the relationship between tinnitus and joint pain from representative samples of Koreans.

Methods. The demographics and the responses to a questionnaire about tinnitus and joint pain severity and mental health status of adults aged $\geq 50$ years in the 2010-2012 Korean National Health and Nutrition Examination Survey were analyzed.

Results. Among 9,032 individuals, $26.7 \%$ reported experiencing tinnitus within the past year. Participants with tinnitus were more frequently older, hearing loss, and had lower education levels, income, and body weight. Participants with regular exercise and sleep had a lower tinnitus prevalence. The incidences of stress, depressed mood, and suicidal ideation were significantly higher in the tinnitus group and participants with joint pain. The rates of participants with tinnitus according to the number of joint pain sites (zero, one, two, and three) was $22.1 \%, 31.4 \%, 33.3 \%$, and $44.2 \%$, and those of participants with severely annoying tinnitus according to the number of joint pain sites (zero, one, two, and three) were $3.3 \%, 6.8 \%, 7.9 \%$, and $10.7 \%$, respectively.

Conclusion. Tinnitus prevalence and severity were significantly related to joint pain, and both conditions were related to psychiatric distress. Thus, the authors suggest that psychiatric distress as a common risk factor for tinnitus and joint pain should be considered when deciding treatment strategies and in guiding public health policy.

Keywords. Tinnitus; Arthralgia; Depression; Anxiety; Comorbidity

\section{INTRODUCTION}

Tinnitus is the perception of sound or noise that results from activity within the nervous system without any real acoustic stimulation within the cochlea [1,2]. One-third of the general population is known to experience tinnitus at least once in their lifetime, with approximately $1 \%-5 \%$ of these patients experienc-

- Received April 11, 2019

Revised August 22, 2019

Accepted September 30, 2019

- Corresponding author: Gi Jung Im

Department of Otolaryngology-Head and Neck Surgery, Korea University

College of Medicine, 73 Inchon-ro, Seongbuk-gu, Seoul 02841, Korea

Tel: +82-2-920-5486, Fax: +82-2-925-5233

E-mail: logopas@korea.ac.kr ing serious psychiatric symptoms [3]. Previous studies reported that tinnitus is associated with psychiatric disorders, including anxiety and depression $[4,5]$, and with a high incidence of depression [6,7]. A systematic review suggested at least three possible associations between depression and tinnitus: depression affects tinnitus, tinnitus predisposes patients to depression, or tinnitus is a comorbidity in patients with depression [8]. Furthermore, the severity of tinnitus has been positively correlated with the severity of depression [9-11].

Joint pain is a common symptom of arthritis. Osteoarthritis $(\mathrm{OA})$ is the most common type of arthritis, mainly reported in the elderly and women, and is classified on the basis of symptoms such as knee joint pain, knee stiffness, hip joint pain, and backache [12]. Recent studies have reported that $\mathrm{OA}$ is associat-

Copyright ( $) 2019$ by Korean Society of Otorhinolaryngology-Head and Neck Surgery.

This is an open-access article distributed under the terms of the Creative Commons Attribution Non-Commercial License (http://creativecommons.org/licenses/by-nc/4.0)

which permits unrestricted non-commercial use, distribution, and reproduction in any medium, provided the original work is properly cited. 
ed with anxiety and depression, as is the case with tinnitus [13]. OA can cause chronic pain and disability, which can lead to poor quality of life and psychiatric disorders.

As mentioned above, anxiety and depression are commonly reported in patients with both tinnitus and OA. A study by McCormack et al. [14] found that patients with tinnitus were at an increased risk of depression (odds ratio [OR], 1.27) and anxiety $(\mathrm{OR}, 1.11)$ symptoms than were those without tinnitus. About $75 \%$ of new patients with tinnitus attribute emotional pressure as the triggering factor rather than ear problems. Subsequently, the triggering factors (i.e., emotional stress, noise, and somatic problems) act synergistically to produce symptomatic tinnitus [15]. In patients with OA, the rate of clinically significant anxiety and/or depression is reported to be $40.7 \%$ [16]. Pain correlated with the Hospital Anxiety and Depression Scale, and disability was greater in patients with depression and/or anxiety [16]. Several hypotheses regarding the relationship between tinnitus and chronic pain have been proposed [17]. Although both tinnitus and chronic pain have many different forms, their pathophysiology and clinical aspects have many similarities [18]. For example, increased auditory sensitivity is one complaint in patients with tinnitus. Auditory sensitivity, also called hyperacusis, is enhanced in individuals with tinnitus than in those without tinnitus, as well as in those with normal audiograms [19].An association between hyperacusis and chronic pain has been proposed, which has been explained by central sensitization. This mechanism can occur after tissue or nerve damage, serving to maintain and enhance nociceptive neurotransmission. This sensitization spreads from the peripheral to the central nervous system, compromising the auditory modulation systems. This leads to the idea that the brainstem and thalamic areas, where multiple sensory pathways converge, are sensitive to chronic pain and are essential for the development of hyperacusis [20]. Various joint pains could be associated with the increase in sensitivity seen in tinnitus. In addition, a previous report documented that central sensitization is related to psychologic disorders such as anxiety and depression [21].

The incidence of tinnitus is high in patients aged over 65 years, with a reported rate of $18.6 \%$ in a nationwide Japanese study [22]. OA affects $33.6 \%$ of adults aged over 65 years in the

\section{H I G H L I G H T S}

- The prevalence of tinnitus significantly increased with increasing numbers of joint pain sites.

- The proportion of participants with severely annoying tinnitus and annoying tinnitus significantly increased with increasing numbers of joint pain sites.

- The prevalence and severity of tinnitus were significantly related to joint pain, and both conditions are thought to share psychiatric distress as a common risk factor.
U.S. [23]. Thus, both tinnitus and OA are common forms of discomfort in the elderly, and both conditions are predicted to occur with increasing age. Accordingly, we hypothesized that tinnitus is associated with joint pain because of common risk factor. Thus, the aim of this study was to investigate the relationship between tinnitus and joint pain from representative samples of Koreans with and without tinnitus based on data from the 2010-2012 Korea National Health and Nutrition Examination Survey (KNHANES), a national population-based cross-sectional survey. In this study, we first analyzed individual risk factors associated with tinnitus and then reported our findings regarding the relationship between tinnitus and various forms of joint pain through the psychiatric processes of stress, depressed mood, and suicidal ideation.

\section{MATERIALS AND METHODS}

\section{Participants}

Data were obtained from the 2010-2012 KNHANES, which is a national population-based cross-sectional survey conducted in the Republic of Korea focused on national health and nutrition state. To exclude somatic tinnitus, individuals with headache, temporomandibular joint (TMJ) pain, tooth pain, cervical pain, and neck pain were excluded. Accordingly, 9,032 participants aged 50 years or older were included in this study. Written informed consent was obtained from all participants, and the Institutional Review Board of Korea University Anam Hospital approved this study (IRB No. ED16185).

\section{Covariates}

In the survey, hearing loss was defined as the averages of frequencies at $0.5,1,2$, and $4 \mathrm{kHz}$ at a pure-tone hearing threshold of a $<25$ decibel hearing level in the ear with worse hearing. Smoking history was classified into three groups: current smoker, ex-smoker, and non-smoker. Alcohol drinking status was also divided into three groups: non-drinkers, mild to moderate drinkers ( $<15 \mathrm{~g} /$ day), and heavy drinkers ( $\geq 15 \mathrm{~g} /$ day) based on the quantity of alcohol consumed per day over the past year. Regular exercise was defined as intense physical activity performed for at least 20 minutes at a time at least three times a week. Body mass index (BMI) was calculated as weight/height ${ }^{2}\left(\mathrm{~kg} / \mathrm{m}^{2}\right)$.

\section{Main variables}

In the KNHANES, the experience of tinnitus was defined as hearing a sound (buzzing, hissing, ringing, humming, roaring, or machinery noise) originating from the ear within the past year. Severity was classified as none, not annoying, annoying, and severely annoying. We defined participants as having joint pain by asking whether they had experienced knee pain, knee stiffness, hip joint pain, and backache for more than 30 days during the preceding 3 months. 
The mental health status survey included questions regarding stress, depressed mood, and suicidal ideation and attempts. Stress was grouped into four categories (none, mild, moderate, and severe). Depressed mood was defined as having experienced a continuous depressed mood that disrupted daily life for 2 or more weeks during the past year. Suicidal ideation was assessed on the basis of a positive answer to the question about suicidal ideation and suicide attempt over the past year.

\section{Statistical analysis}

Statistical analysis was performed using the survey procedure of the SAS ver. 9.3 (SAS Institute Inc., Cary, NC, USA) to account for the complex sampling design and sampling weights of the KNHANES and prescribe nationally representative prevalence estimates. In Table 1, patient characteristics were evaluated using the means and standard errors for continuous variables and percentage and standard errors for categorical variables. The $t$-test and Rao-Scott chi-square test were used to test the difference

Table 1. Analysis of factors associated with tinnitus, including the number of pain sites

\begin{tabular}{|c|c|c|c|}
\hline \multirow{2}{*}{ Variable } & \multicolumn{2}{|c|}{ Tinnitus } & \multirow{2}{*}{$P$-value } \\
\hline & Yes & No & \\
\hline Number & 2,413 & 6,619 & \\
\hline Age (yr) & $64.2 \pm 0.3$ & $61.5 \pm 0.2$ & $<0.001$ \\
\hline Male sex (\%) & $44.4 \pm 1.1$ & $47.1 \pm 0.6$ & 0.05 \\
\hline Hearing loss (>25 dB, \%) & $38.5 \pm 1.3$ & $16.6 \pm 0.6$ & $<0.001$ \\
\hline Smoking (current smoker, \%) & $16.8 \pm 1.1$ & $18.1 \pm 0.6$ & 0.28 \\
\hline Drinking (heavy drinker, \%) & $6.8 \pm 0.7$ & $8.4 \pm 0.5$ & 0.08 \\
\hline Regular exercise (\%) & $16.2 \pm 1.0$ & $18.6 \pm 0.7$ & 0.04 \\
\hline Education ( $\geq$ high school, \%) & $29.6 \pm 1.4$ & $37.3 \pm 1.0$ & $<0.001$ \\
\hline Income (lower quartile, \%) & $36.2 \pm 1.4$ & $25.9 \pm 0.9$ & $<0.001$ \\
\hline Sleep time (hr) & & & 0.001 \\
\hline$<6$ & $23.0 \pm 1.1$ & $20.1 \pm 0.6$ & \\
\hline 6 & $24.0 \pm 1.0$ & $25.5 \pm 0.6$ & \\
\hline 7 & $25.5 \pm 1.0$ & $25.3 \pm 0.7$ & \\
\hline 8 & $18.6 \pm 1.0$ & $22.1 \pm 0.7$ & \\
\hline$>8$ & $8.9 \pm 0.8$ & $7.0 \pm 0.4$ & \\
\hline No. of pain sites & & & $<0.001$ \\
\hline 0 & $55.1 \pm 1.2$ & $68.6 \pm 0.8$ & \\
\hline 1 & $25.1 \pm 1.0$ & $19.3 \pm 0.6$ & \\
\hline 2 & $12.4 \pm 0.8$ & $8.8 \pm 0.4$ & \\
\hline 3 & $7.4 \pm 0.6$ & $3.3 \pm 0.3$ & \\
\hline Moderate to severe stress (\%) & $27.1 \pm 1.0$ & $20.4 \pm 0.6$ & $<0.001$ \\
\hline Depressed mood (\%) & $20.4 \pm 1.0$ & $14.2 \pm 0.5$ & $<0.001$ \\
\hline Suicidal ideation (\%) & $22.7 \pm 1.1$ & $15.3 \pm 0.6$ & $<0.001$ \\
\hline Body mass index $\left(\mathrm{kg} / \mathrm{m}^{2}\right)$ & $23.9 \pm 0.1$ & $24.1 \pm 0.1$ & 0.01 \\
\hline Waist circumference (cm) & $83.4 \pm 0.3$ & $83.6 \pm 0.2$ & 0.33 \\
\hline Vitamin D blood level (ng/mL) & $18.7 \pm 0.2$ & $18.9 \pm 0.2$ & 0.30 \\
\hline
\end{tabular}

Values are presented as mean \pm standard error or percentage of patients \pm standard error. Age, hearing loss, regular exercise, educational level, income, sleep time, number of pain sites, moderate to severe stress, depressed mood, suicidal ideation, and body mass index were significantly associated with tinnitus. according to the presence of tinnitus. In Table 2, psychiatric relationship between tinnitus and pain sites was evaluated using the percentage and standard errors with the Rao-Scott chi-square test. In Table 3, logistic regression methods were used to analyze the association between tinnitus and joint pain or mental health status. The ORs and 95\% confidence intervals (CIs) for tinnitus and joint pain were analyzed.We first described the nonadjusted data (model 1), then the data adjusted for age and sex (model 2), and finally the data adjusted for age, sex, presence of hearing

Table 2. Psychiatric relationship between tinnitus and pain sites

\begin{tabular}{lccc}
\hline Variable & $\begin{array}{c}\text { Stress:moderate } \\
\text { to severe }(\%)\end{array}$ & $\begin{array}{c}\text { Depressed mood } \\
(\%)\end{array}$ & $\begin{array}{c}\text { Suicidal ideation } \\
(\%)\end{array}$ \\
\hline Tinnitus & & & \\
Yes & $27.1 \pm 1.0$ & $20.4 \pm 1.0$ & $22.7 \pm 1.1$ \\
No & $20.4 \pm 0.6$ & $14.2 \pm 0.5$ & $15.3 \pm 0.6$ \\
$P$-value & $<0.001$ & $<0.001$ & $<0.001$ \\
Knee joint pain & & & \\
Yes & $32.5 \pm 1.3$ & $24.1 \pm 1.1$ & $31.2 \pm 1.4$ \\
No & $19.5 \pm 0.5$ & $13.7 \pm 0.5$ & $13.7 \pm 0.5$ \\
$P$-value & $<0.001$ & $<0.001$ & $<0.001$ \\
Knee stiffness & & & \\
Yes & $36.8 \pm 1.8$ & $28.2 \pm 1.8$ & $36.9 \pm 2.0$ \\
No & $20.3 \pm 0.5$ & $14.2 \pm 0.5$ & $14.8 \pm 0.5$ \\
$P$-value & $<0.001$ & $<0.001$ & $<0.001$ \\
Hip joint pain & & & \\
Yes & $35.3 \pm 1.9$ & $29.4 \pm 1.7$ & $34.6 \pm 1.9$ \\
No & $20.8 \pm 0.5$ & $14.3 \pm 0.5$ & $15.4 \pm 0.5$ \\
$P$-value & $<0.001$ & $<0.001$ & $<0.001$ \\
Backache & & & \\
Yes & $32.2 \pm 1.2$ & $23.6 \pm 1.2$ & $31.1 \pm 1.2$ \\
No & $19.1 \pm 0.5$ & $13.4 \pm 0.5$ & $13.0 \pm 0.6$ \\
$P$-value & $<0.001$ & $<0.001$ & $<0.001$ \\
\hline
\end{tabular}

Values are represented as percentage \pm standard error. Tinnitus and these pain sites were significantly related to the percentage of participants with moderate to severe stress, depressed mood, and suicidal ideation.

Table 3. The differences among participants with multiple joint pain sites according to the presence of tinnitus after adjusting for confounding variables

\begin{tabular}{lccc}
\hline \multirow{2}{*}{ Parameter } & \multicolumn{3}{c}{ OR $(95 \% \mathrm{Cl})$} \\
\cline { 2 - 4 } & Model 1 & Model 2 & Model 3 \\
\hline Knee joint pain & $1.72(1.49-1.97)$ & $1.50(1.30-1.74)$ & $1.39(1.19-1.61)$ \\
Knee stiffness & $2.04(1.72-2.41)$ & $1.74(1.46-2.06)$ & $1.61(1.34-1.93)$ \\
Hip joint pain & $1.93(1.63-2.29)$ & $1.72(1.44-2.04)$ & $1.51(1.25-1.81)$ \\
Backache & $1.67(1.46-1.91)$ & $1.47(1.28-1.69)$ & $1.32(1.14-1.53)$ \\
$P$-value & $<0.001$ & $<0.001$ & $<0.001$ \\
\hline
\end{tabular}

The risks for tinnitus were significantly related to knee joint pain, knee stiffness, hip joint pain, and backache. Model 1: non-adjusted; Model 2: adjusted for age and sex; Model 3: adjusted for age, sex, presence of hearing loss, smoking history, alcohol intake, regular exercise, educational level, income, sleep time, stress status, presence of depressed mood, and body mass index.

$\mathrm{OR}$, odds ratio; $\mathrm{Cl}$, confidence interval. 
loss, smoking history, alcohol intake, regular exercise, educational level, income, sleep time, stress status, presence of depressed mood, and BMI (model 3).

\section{RESULTS}

\section{Individual risk factors associated with tinnitus}

The characteristics of participants according to the presence of tinnitus are shown in Table 1. Among 9,032 selected individuals, 2,413 reported having experienced tinnitus within the preceding 12 months (26.7\%, tinnitus group). The mean age of the tinnitus group was significantly higher than that of the non-tinnitus group ( $64.2 \pm 0.3$ vs. $61.5 \pm 0.2$ years; $P<0.001)$. The prevalence of hearing loss was significantly higher in the tinnitus group than the non-tinnitus group $(38.5 \% \pm 1.3 \%$ vs. $16.6 \% \pm 0.6 \%$; $P$ $0.001)$. No significant differences were observed between the two groups in sex or smoking and drinking history (Table 1).

The proportion of participants with an education level $\geq$ high school was significantly lower in the tinnitus group than in the non-tinnitus group $(29.6 \% \pm 1.4 \%$ vs. $37.3 \% \pm 1 \% ; P<0.001)$, whereas the proportion of participants within the lower quartile of household income was significantly higher in the tinnitus group than in the non-tinnitus group $(36.2 \% \pm 1.4 \%$ vs. $25.9 \% \pm 0.9 \%$; $P<0.001)$. The proportion of participants with regular exercise was also significantly lower in the tinnitus group than in the nontinnitus group $(16.2 \% \pm 1 \%$ vs. $18.6 \% \pm 0.7 \% ; P=0.037)$. Sleep time was significantly associated with tinnitus $(P=0.001)$ and displayed a U-shaped relationship.

The percentage of participants with moderate to severe stress was significantly higher in the tinnitus group than in the nontinnitus group $(27.1 \% \pm 1 \%$ vs. $20.4 \% \pm 0.6 \% ; P<0.001)$, as was the proportion of participants with depressed mood ( $20.4 \% \pm 1 \%$ vs. $14.2 \% \pm 0.5 \% ; P<0.001)$ and suicidal ideation

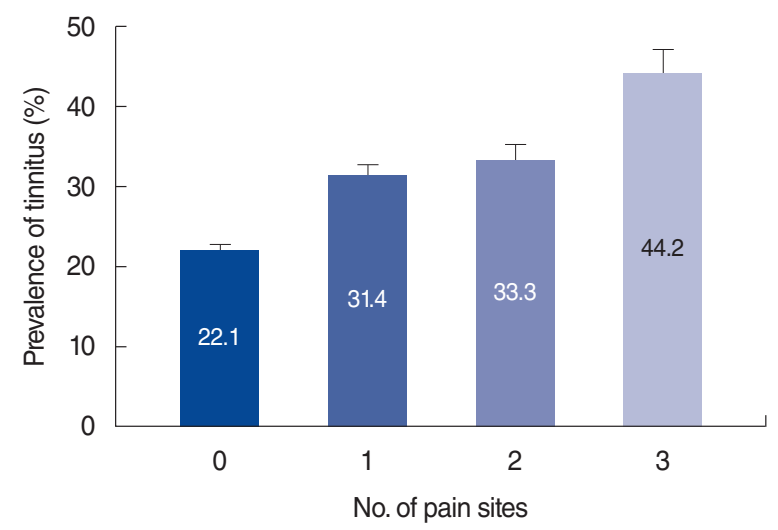

Fig. 1. Prevalence of tinnitus according to the number of joint pain sites. The prevalence of tinnitus significantly increased with increasing numbers of joint pain sites ( $P$ for trend $<0.001)$.
$(22.7 \% \pm 1.1 \%$ vs. $15.3 \% \pm 0.6 \% ; P<0.001)$. The mean BMI was significantly lower in the tinnitus group than in the non-tinnitus group ( $\left.23.9 \pm 0.1 \mathrm{vs.} 24.1 \pm 0.1 \mathrm{~kg} / \mathrm{m}^{2} ; P=0.01\right)$. No significant intergroup differences were observed in waist circumference and plasma vitamin $\mathrm{D}$ levels.

\section{Relation between tinnitus and joint pain}

As shown in Table 1, the proportion of participants with joint pain was higher in the tinnitus group than in the non-tinnitus group $(P<0.001)$. As seen in Table 2 , the presence of tinnitus and pain at the four sites investigated were significantly related to moderate to severe stress, depressed mood, and suicidal ideation (all $P<0.001$ ). Table 3 presents the differences among participants with multiple joint pain sites according to the presence of tinnitus after adjusting for confounding variables. In model 1, the risk for tinnitus was significantly related to knee joint pain (OR, 1.72; 95\% CI, 1.49-1.97), knee stiffness (OR, 2.04; 95\% CI, 1.72-2.41), hip joint pain (OR, 1.93; 95\% CI, 1.63-2.29), and backache (OR, 1.67; 95\% CI, 1.46-1.91). In model 2, the risk for tinnitus was significantly related to knee joint pain (OR, 1.50; 95\% CI, 1.30-1.74), knee stiffness (OR, 1.74; 95\% CI, 1.46-2.06), hip joint pain (OR, 1.72; 95\% CI, 1.44-2.04), and backache (OR, 1.47; 95\% CI, 1.28-1.69). In model 3, the risk for tinnitus was also significantly related to knee joint pain (OR, 1.39; 95\% CI, 1.19-1.61), knee stiffness (OR, 1.61; 95\% CI, 1.34-1.93), hip joint pain (OR, 1.51; 95\% CI, 1.25-1.81), and backache (OR, 1.32; 95\% CI, 1.14-1.53).

\section{Prevalence of tinnitus according to the number of joint pain sites}

Fig. 1 presents the rate of tinnitus as a function of the number of joint pain sites. The proportion of participants with tinnitus according to the number of joint pain sites (zero, one, two, and three) was $22.1 \% \pm 0.7 \%, 31.4 \% \pm 1.3 \%, 33.3 \% \pm 2 \%$, and

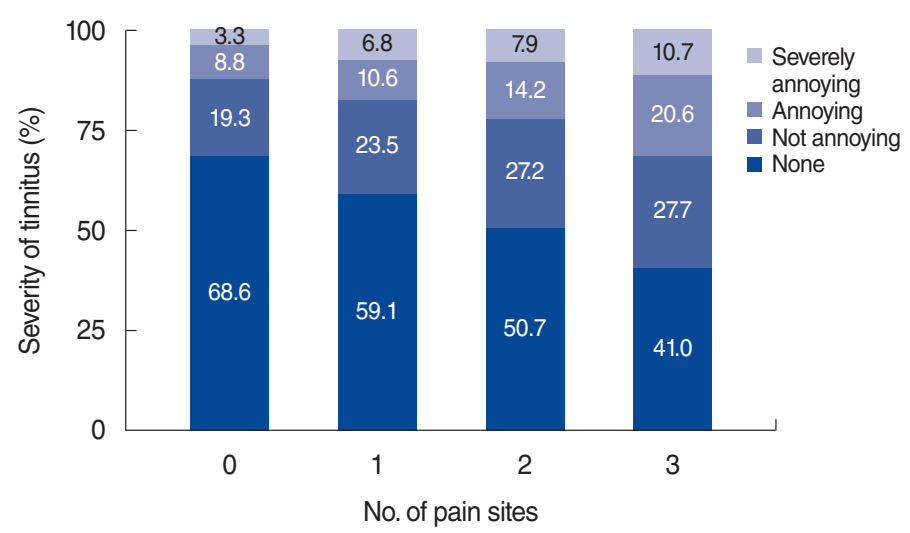

Fig. 2. Severity of tinnitus according to the number of joint pain sites. The severity of tinnitus was classified as none, not annoying, annoying, and severely annoying. The proportion of participants with severely annoying tinnitus and annoying tinnitus significantly increased with increasing numbers of joint pain sites ( $P$ for trend $<0.001$ ). 
$44.2 \% \pm 3 \%$, respectively. The rate of tinnitus was found to significantly increase according to the number of joint pain sites $(P$ for trend $<0.001$ ).

\section{Severity of tinnitus according to the number of joint pain sites}

Fig. 2 presents the severity of tinnitus according to the number of joint pain sites. The severity of tinnitus was classified as none, not annoying, annoying, and severely annoying. The proportion of participants with severely annoying tinnitus according to the number of joint pain sites (zero, one, two, and three) was 3.3\%, $6.8 \%, 7.9 \%$, and $10.7 \%$, respectively; the corresponding rates for annoying tinnitus were $8.8 \%, 10.6 \%, 14.2 \%$, and $20.6 \%$, respectively. The proportion of participants with annoying tinnitus and severely annoying tinnitus significantly increased with an increasing number of joint pain sites ( $P$ for trend $<0.001$ ).

\section{DISCUSSION}

To our knowledge, a comprehensive analysis of the comorbidity of tinnitus and joint pain is absent in the literature. However, numerous reports in the literature have documented tinnitus and temporomandibular disorder, headache, and facial pain $[24,25]$. Thus, this could be the first study that statistically evaluates the comorbidity of tinnitus and various sites of joint pain. The first objective of this study was achieved, and tinnitus was found to be significantly associated with the number of joint pain sites. Tinnitus and joint pain were significantly associated with stress, depressed mood, and suicidal ideation.

A previous cross-sectional study reported that knee joint pain is a factor associated with tinnitus in elderly Japanese patients. According to that study, not only knee joint pain, but hearing difficulty, depressed mood, prescribed medication, and coronary heart disease were factors associated with tinnitus [22]. The association of tinnitus with psychiatric disorders such as anxiety and depression has been well described [26-28], as has been chronic pain, including joint pain [29]. Therefore, actively treating psychiatric disorders that may be present in patients with tinnitus and joint pain is very important.

Various studies have reported on the relationship between tinnitus and chronic pain. Moller [18] proposed two hypotheses to explain the similarities observed between tinnitus and chronic pain: the pathogenesis of the peripheral nervous system can explain the symptoms or the symptoms can be caused by changes in central nervous system function. Furthermore, the author proposed that tinnitus and chronic pain are caused by modulation of the nervous system that occurs as a result of neural plasticity. Neural plasticity is thought to underlie the generation of tinnitus. Bartels et al. [30] described that an altered afferent signal to the peripheral auditory system results in the generation of tinnitus in the central auditory system, and that neural plasticity can be divided into initiation of the generation of new connections through axonal sprouting (early modifications) and lateral spread of neural activity and development of excitatory regions of the central nervous system (late modifications). Chronic pain has also been attributed to neural plasticity in the primary afferent nociceptive nerve pathway [31] and brain regions such as the hippocampus [32].

Central sensitization is one mechanism that can explain the relationship between tinnitus and joint pain. Central sensitization enhances the function of neurons and circuits in the nociceptive pathway owing to increased membrane excitability and synaptic potency, and suppresses inhibition. This is an expression of neural plasticity of the sensory nervous system in response to activity, inflammation, and nerve damage [33]. Additionally, several authors reported that central sensitization is related to psychiatric disorders, such as anxiety and depression [21]. Owing to central sensitization, neurons in the auditory modulation system may undergo changes similar to central neuropathic pain, and these changes may produce hyperactivity, resulting in tinnitus [34].

We considered that the presence of somatic tinnitus could affect the results of this study. In some cases, tinnitus can be generated or modulated by inputs from somatosensory systems, and this is evidence of the interactions between auditory and somatosensory signals generated early in the auditory pathways [35]. Somatic tinnitus mainly manifests as a somatic stimulation associated with the neck, jaw, extraocular muscles, and the TMJ. Modulation of the firing rate and synchrony in the dorsal cochlear nucleus due to somatic stimulations have been reported as the cause of somatic tinnitus [35]. Thus, individuals with headache, TMJ pain, tooth pain, cervical pain, and neck pain were excluded from this study to rule out somatic tinnitus.

The present study showed that among Koreans aged 50 years or older, approximately one in four had tinnitus. This rate was similar to that previously reported in a few community-based studies in aged populations [22]. In our analysis, tinnitus occurred in the elderly. However, some authors proposed that the rate of tinnitus increased with age until 60-69 years, after which it declined [36]. One likely explanation for this inverse relationship is that tinnitus may be related to other factors that confer a selective mortality disadvantage among patients. The possibility also exists, however, that late improvement of symptoms may be considered part of the natural history of tinnitus [36]. Hearing loss is known to be the most significant risk factor of tinnitus [1]. As expected, the tinnitus group had a significantly higher prevalence of hearing loss than the non-tinnitus group in this study. However, the authors found a significant relationship between tinnitus and joint pain from the multivariate logistic regression analysis that adjusted related variables including hearing loss.

Our results demonstrate the relationship between tinnitus and lifestyle, as both exercise and sleep were associated with a low incidence of tinnitus. A healthy lifestyle is considered an important factor for a healthy mental status. Therefore, a healthy life- 
style has some benefit in reducing tinnitus-related stress. In contrast, tinnitus was found to be more commonly associated with the inability to sleep, probably since the perceived tinnitus would increase annoyance, anxiety, and stress reactions [37]. In this study, participants who usually sleep for less than 6 hours or more than 8 hours showed a higher prevalence of tinnitus. However, because of the limitations associated with a cross-sectional study, the association between tinnitus and sleep disturbance was unclear. Additionally, a low socioeconomic status (SES) was found to be associated with a higher prevalence of tinnitus. Studies have demonstrated that a relationship exists between SES, hearing impairment, and tinnitus [38,39]. Prolonged periods of occupational noise exposure, positive attitudes towards noise, and non-use of hearing protection are postulated as possible causes. Tinnitus occurred more frequently in individuals who had a lower BMI in this study. However, our results differ from that of a previous study in the U.S., in which participants with a higher BMI had a higher prevalence of tinnitus [36]. Data on the association between the BMI and tinnitus are limited and require further investigation.

The strength of this study is its sample size, which is representative of the general population from which it was drawn. Nevertheless, several limitations of this study should be considered. First, using a cross-sectional study makes causative relationships difficult to establish. Second, questions about tinnitus, joint pain, and mental health status in the KNHANES were simple and brief, and hence, misclassification of statuses might have occurred.

In conclusion, this study showed that the prevalence and severity of tinnitus was significantly related to joint pain, and that both conditions were significantly related to stress, depressed mood, and suicidal ideation. Thus, we suggest that tinnitus and joint pain may be linked through common mechanisms of psychiatric states, and that the psychiatric distress as a common risk factor for tinnitus and joint pain is should be considered when deciding treatment strategies and in guiding public health policy.

\section{CONFLICT OF INTEREST}

No potential conflict of interest relevant to this article was reported.

\section{ACKNOWLEDGMENTS}

This research was supported by the Basic Science Research Program through the National Research Foundation of Korea (NRF) funded by Korea Evaluation Institute of Industrial Technology under Grant R1621961; Korea Health Industry Development Institute under Grant R1606511; and Korea University Research Grants K1609821 and Q1620561.

We thank the doctors, residents, and administration of the training hospitals involved in this survey, as well as the Division of Chronic Disease Surveillance of the Korea Centers for Disease Control \& Prevention.

\section{ORCID}

$\begin{array}{ll}\text { Euyhyun Park } & \text { https://orcid.org/0000-0003-4373-6942 } \\ \text { Hyunjung Kim } & \text { https://orcid.org/0000-0002-8815-5941 } \\ \text { In Hak Choi } & \text { https://orcid.org/0000-0001-7280-7995 } \\ \text { Hye Min Han } & \text { https://orcid.org/0000-0002-5612-1261 } \\ \text { Kyungdo Han } & \text { https://orcid.org/0000-0002-6096-1263 } \\ \text { Hak Hyun Jung } & \text { https://orcid.org/0000-0002-4402-1132 } \\ \text { Gi Jung Im } & \text { https://orcid.org/0000-0002-9457-4253 }\end{array}$

\section{AUTHOR CONTRIBUTIONS}

Conceptualization: KH, GJI. Data curation: KH, GJI. Formal analysis: KH, EP. Funding acquisition: GJI. Methodology: KH, GJI. Project administration: HK, IHC, HMH, GJ, EP. Visualization: HHJ. Writing-original draft: EP, GJI. Writing-review \& editing: all authors.

\section{REFERENCES}

1. Jastreboff PJ. Phantom auditory perception (tinnitus): mechanisms of generation and perception. Neurosci Res. 1990 Aug;8(4):221-54.

2. Seo JH, Kang JM, Hwang SH, Han KD, Joo YH. Relationship between tinnitus and suicidal behaviour in Korean men and women: a crosssectional study. Clin Otolaryngol. 2016 Jun;41(3):222-7.

3. Martines F, Bentivegna D, Di Piazza F, Martines E, Sciacca V, Martinciglio G. Investigation of tinnitus patients in Italy: clinical and audiological characteristics. Int J Otolaryngol. 2010;2010:265861.

4. Reynolds P, Gardner D, Lee R. Tinnitus and psychological morbidity: a cross-sectional study to investigate psychological morbidity in tinnitus patients and its relationship with severity of symptoms and illness perceptions. Clin Otolaryngol Allied Sci. 2004 Dec;29(6):62834.

5. Adoga AA, Adoga AS, Obindo JT.Tinnitus and the prevalence of comorbid psychological stress. Niger J Med. 2008 Jan-Mar;17(1):95-7.

6. Pinto PC, Marcelos CM, Mezzasalma MA, Osterne FJ, de Melo Tavares de Lima MA, Nardi AE. Tinnitus and its association with psychiatric disorders: systematic review. J Laryngol Otol. 2014 Aug; 128(8):660-4.

7. Zoger S, Svedlund J, Holgers KM. Relationship between tinnitus severity and psychiatric disorders. Psychosomatics. 2006 Jul-Aug;47(4): 282-8.

8. Geocze L, Mucci S, Abranches DC, Marco MA, Penido Nde O. Systematic review on the evidences of an association between tinnitus and depression. Braz J Otorhinolaryngol. 2013 Jan-Feb;79(1):10611.

9. Andersson G, Vretblad P, Larsen HC, Lyttkens L. Longitudinal follow-up of tinnitus complaints. Arch Otolaryngol Head Neck Surg. 2001 Feb;127(2):175-9.

10. Holgers KM, Erlandsson SI, Barrenas ML. Predictive factors for the severity of tinnitus. Audiology. 2000 Sep-Oct;39(5):284-91. 
11. Folmer RL. Long-term reductions in tinnitus severity. BMC Ear Nose Throat Disord. 2002 Sep 16;2(1):3.

12. Timmermans EJ, Schaap LA, Herbolsheimer F, Dennison EM, Maggi $\mathrm{S}$, Pedersen NL, et al. The influence of weather conditions on joint pain in older people with osteoarthritis: results from the European Project on OSteoArthritis. J Rheumatol. 2015 Oct;42(10):1885-92.

13. Sharma A, Kudesia P, Shi Q, Gandhi R. Anxiety and depression in patients with osteoarthritis: impact and management challenges. Open Access Rheumatol. 2016 Oct;8:103-13.

14. McCormack A, Edmondson-Jones M, Fortnum H, Dawes PD, Middleton $\mathrm{H}$, Munro KJ, et al. Investigating the association between tinnitus severity and symptoms of depression and anxiety, while controlling for neuroticism, in a large middle-aged UK population. Int $\mathrm{J}$ Audiol. 2015;54(9):599-604.

15. Han BI, Lee HW, Kim TY, Lim JS, Shin KS. Tinnitus: characteristics, causes, mechanisms, and treatments. J Clin Neurol. 2009 Mar;5(1): 11-9.

16. Axford J, ButtA, Heron C, Hammond J, Morgan J,AlaviA, et al. Prevalence of anxiety and depression in osteoarthritis: use of the Hospital Anxiety and Depression Scale as a screening tool. Clin Rheumatol. 2010 Nov;29(11):1277-83.

17. Tonndorf J. The analogy between tinnitus and pain: a suggestion for a physiological basis of chronic tinnitus. Hear Res. 1987;28(2-3): 271-5.

18. Moller AR. Similarities between severe tinnitus and chronic pain. J Am Acad Audiol. 2000 Mar;11(3):115-24.

19. Hebert S, Fournier P, Norena A. The auditory sensitivity is increased in tinnitus ears. J Neurosci. 2013 Feb;33(6):2356-64.

20. Suhnan AP, Finch PM, Drummond PD. Hyperacusis in chronic pain: neural interactions between the auditory and nociceptive systems. Int J Audiol. 2017 Nov;56(11):801-9.

21. Schlereth T, Heiland A, Breimhorst M, Fechir M, Kern U, Magerl W, et al. Association between pain, central sensitization and anxiety in postherpetic neuralgia. Eur J Pain. 2015 Feb;19(2):193-201.

22. Michikawa T, Nishiwaki Y, Kikuchi Y, Saito H, Mizutari K, Okamoto $\mathrm{M}$, et al. Prevalence and factors associated with tinnitus: a community-based study of Japanese elders. J Epidemiol. 2010;20(4):271-6.

23. Lawrence RC, Felson DT, Helmick CG, Arnold LM, Choi H, Deyo RA, et al. Estimates of the prevalence of arthritis and other rheumatic conditions in the United States. Part II. Arthritis Rheum. 2008 Jan;58(1):26-35.

24. Upton LG, Wijeyesakere SJ.The incidence of tinnitus in people with disorders of the temporomandibular joint. IntTinnitus J. 2004;10(2): 174-6.

25. Pezzoli M, Ugolini A, Rota E, Ferrero L, Milani C, Pezzoli L, et al. Tinnitus and its relationship with muscle tenderness in patients with headache and facial pain. J Laryngol Otol. 2015 Jul;129(7):638-43.

26. Halford JB, Anderson SD. Anxiety and depression in tinnitus sufferers. J Psychosom Res. 1991;35(4-5):383-90.

27. Bhatt JM, Bhattacharyya N, Lin HW. Relationships between tinnitus and the prevalence of anxiety and depression. Laryngoscope. 2017 Feb;127(2):466-9.

28. Hebert S, Canlon B, Hasson D, Magnusson Hanson LL, Westerlund $\mathrm{H}$, Theorell T. Tinnitus severity is reduced with reduction of depressive mood: a prospective population study in Sweden. PLoS One. 2012;7(5):e37733.

29. Bair MJ, Robinson RL, Katon W, Kroenke K. Depression and pain comorbidity: a literature review.Arch Intern Med. 2003 Nov;163(20): 2433-45.

30. Bartels H, Staal MJ, Albers FW. Tinnitus and neural plasticity of the brain. Otol Neurotol. 2007 Feb;28(2):178-84.

31. Reichling DB, Levine JD. Critical role of nociceptor plasticity in chronic pain. Trends Neurosci. 2009 Dec;32(12):611-8.

32. McEwen BS. Plasticity of the hippocampus: adaptation to chronic stress and allostatic load. Ann NYAcad Sci. 2001 Mar;933:265-77.

33. Latremoliere A, Woolf CJ. Central sensitization: a generator of pain hypersensitivity by central neural plasticity. J Pain. 2009 Sep;10(9): 895-926.

34. Moller AR. Tinnitus and pain. Prog Brain Res. 2007;166:47-53.

35. Shore S, Zhou J, Koehler S. Neural mechanisms underlying somatic tinnitus. Prog Brain Res. 2007;166:107-23.

36. Shargorodsky J, Curhan GC, Farwell WR. Prevalence and characteristics of tinnitus among US adults. Am J Med. 2010 Aug;123(8): 711-8.

37. Hasson D, Theorell T, Wallen MB, Leineweber C, Canlon B. Stress and prevalence of hearing problems in the Swedish working population. BMC Public Health. 2011 Feb;11:130.

38. Berg AL, Serpanos YC. High frequency hearing sensitivity in adolescent females of a lower socioeconomic status over a period of 24 years (1985-2008). J Adolesc Health. 2011 Feb;48(2):203-8.

39. Widen SE, Erlandsson SI. The influence of socio-economic status on adolescent attitude to social noise and hearing protection. Noise Health. 2004 Oct-Dec;7(25):59-70. 